\title{
Optimal Unified Architectures for the Real-Time Computation of Time-Recursive Discrete Sinusoidal Transforms
}

\author{
K. J. R. Liu, Member, IEEE, C. T. Chiu, Member, IEEE, R. \\ K. Kolagotla, Member, IEEE, and J. F. JáJá, Senior Member, IEEE
}

\begin{abstract}
An optimal unified architecture that can efficiently compute the Discrete Cosine, Sine, Hartley, Fourier, Lapped Orthogonal, and Complex Lapped transforms for a continuous stream of input data that arise in signal/image communications is proposed. This structure uses only half as many multipliers as the previous best known scheme [1]. The proposed architecture is regular, modular, and has only local interconnections in both data and control paths. There is no limitation on the transform size $N$ and only $2 N-2$ multipliers are needed for the DCT. The throughput of this scheme is one input sample per clock cycle. We provide a theoretical justification by showing that any discrete transform whose basis functions satisfy the Fundamental Recurrence Formula has a second-order autoregressive structure in its filter realization. We also demonstrate that dual generation transform pairs share the same autoregressive structure. We extend these time-recursive concepts to multi-dimensional transforms. The resulting $d$-dimensional structures are fully-pipelined and consist of only $d$ 1-D transform arrays and shift registers.
\end{abstract}

\section{INTRODUCTION}

D ISCRETE sinusoidal transforms play significant roles in various digital signal processing applications, such as spectrum analysis, image and speech signal processing, computer tomography, data compression, and signal reconstruction [2]-[5]. Among different discrete sinusoidal transforms, the discrete cosine transform (DCT) [6]-[9], the discrete sine transform (DST) [9], [10], the discrete Hartley transform (DHT) [27], [28], [26], [6], and the discrete Fourier transform (DFT) [3] are widely used because of their efficient performance [5], [20]-[24]. Recently, the Lapped Orthogonal Transform (LOT) [14], and the Complex Lapped transform (CLT) [13] were introduced for transform coding with significantly reduced blocking effects and for motion estimation.

In real-time signal processing applications, especially in speech/image communications and radar/sonar signal processing, input data arrive serially. In traditional FFT based algorithms, the serial data is buffered and then transformed using the FFT scheme of complexity $O(N \log N)$ [3]. Buffering the serial data requires $O(N)$ time. In this paper, we describe

Manuscript received April 13, 1993; revised October 18, 1993.

The authors are with the Electrical Engineering Dept., Systems Research Center, University of Maryland, College Park, MD 20742.

R. K. Kolagatla is now with IBM Corp., Essex Junction, VT 05452

C. T. Chiu is now with National Chung Cheng University, Taiwan.

This work was partially supported by the NSF grant ECD-8803012, the NSF grant MIP9309506, the ONR grant N00014-93-1-0566, and the State of Maryland MIPS/Micro Star Co.

IEEE Log Number 9400883. a novel architecture that merges the buffering and transform operations into a single unit of total hardware complexity $O(N)$, and the $O(N)$ waiting time is thus eliminated. Unlike the FFT, this architecture has only local interconnections and is better suited for VLSI implementations. It is important to note that the proposed architectures generate time-recursive transforms, not just block transforms, i.e., the transform of the $N$ points $[x(t+1), x(t+2), \cdots, x(t+N)]$ is generated one clock cycle after the transform of $[x(t), x(t+1), \cdots, x(t+N-$ $1)]$ is generated. To generate time-recursive transforms, the traditional fast algorithms based architectures require $O(\log N)$ time using $O(N \log N)$ hardware, while the architectures we propose require only a constant time with $O(N)$ hardware. Time-recursive transforms are currently gaining widespread use in motion estimation, in video signal processing, and in reducing blocking effects in data compression.

We have shown in [1] that when discrete transforms are performed on segments of a continuously incoming data stream, transforms can be realized by a unified lattice structure with a data throughput rate of one input sample per clock cycle. This architecture is regular, modular, and free of global interconnections. Unlike the many fast algorithms for DFT, DCT, and DHT, there is no constraint on the transform size $N$. Table I [1] summarizes a comparison of the time-recursive approach with other well-known fast algorithms. A timerecursive lattice 2-D DCT structure with applications to the HDTV systems is also given in [17]. This 2-D DCT structure requires only two 1-D DCT blocks and is fully-pipelined with no transposition.

In this paper, we describe an optimal unified filter structure, which preserves the advantages of the lattice architecture, while reducing the hardware complexity in half. In the timerecursive lattice architecture, two transforms called the dual generated pairs, are obtained simultaneously. The unified filter structure is more suitable for applications where only one transform is required. We develop a systematic approach to derive the time-recursive unified filter architecture for any discrete transform. We show that all the resulting unified filter architectures have a similar second-order autoregressive structure with minimum number of multipliers. A theoretical basis for this fact is provided. We also demonstrate that the time-recursive concept can be generalized to multidimensional transforms by using only the one-dimensional transform architecture and simple shift registers. An area-time 
TABLE 1

COMParisons OF DifFERENT DCT ALGORITHMS

\begin{tabular}{llllll}
\hline & Liu-Chiu1 & Liu-Chiu2 & Chen [23] et al. & Lee [24] \\
\hline No. of Multipliers & $6 N-4$ & $4 N$ & $N \ln (N)-3 N / 2+4$ & $(N / 2) \ln (N)$ \\
latency & $N$ & $2 N$ & $N / 2$ & {$[\ln (N)(\ln (N)-1)] / 2 \quad 3 N / 2($ order $)$} & power of 2 \\
limitation on transform size $N$ & no & no & power of 2 & global & power of 2 \\
communication & local & local & global & global \\
I/O operation & $S I P O$ & $S I S O$ & $P I P O$ & $S I P O$ \\
\hline
\end{tabular}

complexity analysis is also provided to show that the proposed approach is asymptotically optimal in speed and area.

The rest of this paper is organized as follows. The unified lattice structure for sinusoidal transforms is summarized in Section II. The derivation of the optimal unified filter structure from the transfer function of the discrete sinusoidal transforms is discussed in Section III. The architectures of the inverse discrete sinusoidal transforms based on the IIR filter realization are presented in Section IV. In Section V, the characteristics of these architectures are discussed from a theoretical point of view. The unified architectures for time-recursive based multi-dimensional discrete sinusoidal transforms are discussed in Section VI. Finally, we give a conclusion in Section VII.

\section{LATTICE STRUCTURE FOR DISCRETE SINUSOIDAL TRANSFORMS}

The time-recursive approach has been shown to be efficient in both hardware and computational complexity for the computation of discrete sinusoidal transforms (DXT), (such as the DCT, DST, and DHT), for time series input data stream [1]. In this section, we will summary and provide a unified view of lattice structures for time-recursive approach.

Denote the discrete sinusoidal transform DXT of a data sequence of length $N[x(t), x(t+1), \cdots, x(t+N-1) ; t=$ $0,1,2, \cdots]$ at time $t$ as

$$
\begin{aligned}
X(k, t)=C(k) \sum_{n=t}^{t+N-1} x(n) P_{n-t}(k), & \\
k & =0,1, \cdots, N-1,
\end{aligned}
$$

where $P_{n-t}(k)$ are transform basis functions and $C(k)$ are constants used for normalization. It was shown in [1] that most discrete sinusoidal transforms have dual generated pairs. That is, the lattice structure used for generating one transform automatically generates its dual. For example, the dual of the DCT is the DST. Both the transform and its dual have similar updating relations. Let us denote the dual generated pairs by $X_{x c}(k, t)$ and $X_{x s}(k, t)$. Then, the time-recursive relation between $X_{x}(k, t)$ and $X_{x}(k, t+1)$ can be obtained by eliminating the effect of the first term of the previous sequence and updating the effect of the last term of the current sequence. In general, the dual generation properties between the transform pairs $X_{x c}(k, t)$ and $X_{x s}(k, t)$ are given by the following equations $[1]$ :

$$
\begin{aligned}
& X_{x c}(k, t+1) \\
& =e(k)\left\{\left[X_{x c}(k, t)+\left[x(t+N)(-1)^{k}-x(t)\right] D_{c}\right] \Gamma_{c}\right. \\
& \left.\quad+\left[X_{x s}(k, t)+\left[x(t+N)(-1)^{k}-x(t)\right] D_{s}\right] \Gamma_{s}\right\}
\end{aligned}
$$

and

$$
\begin{aligned}
& X_{x s}(k, t+1) \\
& =f(k)\left\{\left[X_{x s}(k, t)+\left[x(t+N)(-1)^{k}-x(t)\right] D_{s}\right] \Gamma_{c}\right. \\
& \left.\quad-\left[X_{x c}(k, t)+\left[x(t+N)(-1)^{k}-x(t)\right] D_{c}\right] \Gamma_{s}\right\},
\end{aligned}
$$

where $D_{c}$ and $D_{s}$ are the associated cos and $\sin$ transform kernels of the DXT with fixed index $n$. Coefficients $e(k)$ and $f(k)$ depend on the definition of the transforms and are always equal to one except for the two transforms LOT and CLT. Here, we will briefly describe the definition of the various discrete sinusoidal transforms [10]-[13].

The one-dimensional (1-D) DCT of an input data sequence $[x(t), x(t+1), \cdots, x(t+N-1), t=0,1,2, \cdots]$ is defined as [11]

$$
\begin{array}{r}
X_{c}(k, t)=C(k) \sqrt{\frac{2}{N}} \sum_{n=t}^{t+N-1} x(n) \cos \left[\left(n-t+\frac{1}{2}\right) \frac{k \pi}{N}\right], \\
k=0,1, \cdots, N-1,
\end{array}
$$

and the 1-D DST is defined as [10]

$$
\begin{gathered}
X_{s}(k, t)=C(k) \sqrt{\frac{2}{N}} \sum_{n=t}^{t+N-1} x(n) \sin \left[\left(n-t+\frac{1}{2}\right) \frac{k \pi}{N}\right], \\
k=1, \cdots, N
\end{gathered}
$$

where

$$
C(k)= \begin{cases}\frac{1}{\sqrt{2}} & \text { if } k=0 \text { or } N \\ 1 & \text { otherwise. }\end{cases}
$$

The definition of DHT is given by [12]

$$
\begin{aligned}
& X_{h}(k, t)=\frac{1}{\sqrt{N}} \sum_{n=t}^{t+N-1} x(n) \operatorname{cas}\left(2(n-t) \frac{\pi k}{N}\right) \\
& k=0,1, \cdots, N-1,
\end{aligned}
$$

where $\operatorname{cas} \theta \triangleq \cos \theta+\sin \theta$.

The Discrete Fourier Transform (DFT) is defined as [3]

$$
\begin{array}{r}
X_{f}(k, t)=\frac{1}{\sqrt{N}} \sum_{n=t}^{t+N-1} x(n) \exp \left\{-j 2(n-t) \frac{\pi k}{N}\right\} \\
k=0,1, \cdots, N-1 .
\end{array}
$$

The Lapped Orthogonal Transform (LOT) [14], [13] of $2 N$ samples $\left[x\left(t-N+\frac{1}{2}\right), x\left(t-N+\frac{3}{2}\right), \cdots, x\left(t+N-\frac{1}{2}\right)\right]$ is 
TABLE II

Coefficients of the LATtice Structlre for the DXT

\begin{tabular}{|c|c|c|c|c|c|c|}
\hline & $\Gamma_{C}$ & $\Gamma_{s}$ & $D_{c}$ & $D_{s}$ & $e(k)$ & $f(k)$ \\
\hline DCT/DST & $\cos (\pi k / N)$ & $\sin (\pi k / N)$ & $\begin{array}{l}C(k) \sqrt{\frac{2}{N}} \\
\cdot \cos (\pi k / 2 N)\end{array}$ & $\begin{array}{l}C(k) \sqrt{\frac{2}{N}} \\
\cdot \sin (\pi k / 2 N)\end{array}$ & 1 & 1 \\
\hline DHT/DFT & $\cos (2 \pi k / N)$ & $\sin (2 \pi k / N)$ & $\sqrt{\frac{1}{8}}$ & 0 & 1 & 1 \\
\hline LOT/CLT & $\cos (\pi / 2 N)$ & $\sin (\pi / 2 N)$ & $\begin{array}{l}\sqrt{\frac{1}{N}}(-1)^{k} j \cdot \exp \left(-j \theta_{k}\right) \\
\cdot \sin (\pi / 4 N)\end{array}$ & $\begin{array}{l}\sqrt{\frac{1}{N}}(-1)^{k} j \\
\exp \left(-j \theta_{k}\right) \\
\cdot \sin (\pi / 4 N)\end{array}$ & $\exp \left(j 2 \theta_{k}\right)$ & $\exp \left(j 2 \theta_{k}\right)$ \\
\hline
\end{tabular}

defined as

$$
\begin{aligned}
& X_{\text {lot }}(k, t) \\
& =\left\{\begin{array}{cc}
\sqrt{\frac{2}{N}} \sum_{n=t-\left(N-\frac{1}{2}\right)}^{t+\left(N-\frac{1}{2}\right)} & x(n) \cos \frac{(2 k+1)(n-t) \pi}{2 N} \\
& \cdot \cos \frac{(n-t) \pi}{2 N}+\alpha_{k}, \\
& k=0,2, \cdots,(N-2), \\
& \text { even part of the CLT } \\
\sqrt{\frac{2}{N}} \sum_{n=t-\left(N-\frac{1}{2}\right)}^{t+\left(N-\frac{1}{2}\right)} & x(n) \sin \frac{(2 k+1)(n-t) \pi}{2 N} \\
& \cdot \cos \frac{(n-t) \pi}{2 N}+\beta_{n k}, \\
& k=1,3, \cdots,(N-1), \\
& \text { odd part of the CLT }
\end{array}\right.
\end{aligned}
$$

where $\alpha_{k}=\beta_{n k}=0$, except for $\alpha_{0}=-(\sqrt{2}-1) /(2 \sqrt{2})$, and $\beta_{n(N-1)}=(-1)^{n+\frac{1}{2}} \alpha_{0}$.

The Complex Lapped Transform (CLT) [13] of $2 N$ samples $\left[x\left(t-N+\frac{1}{2}\right), x\left(t-N+\frac{3}{2}\right), \cdots, x\left(x+N-\frac{1}{2}\right)\right]$ is defined as

$$
\begin{aligned}
X_{c i t}(k, t)= & \frac{1}{\sqrt{N}} \sum_{n=t-\left(N-\frac{1}{2}\right)}^{t+\left(N-\frac{1}{2}\right)} x(n) \\
& \cdot \exp \left\{-j \frac{(2 k+1)(n-t) \pi}{2 N}\right\} \cos \frac{(n-t) \pi}{2 N}, \\
k & =0,1, \cdots, N-1 .
\end{aligned}
$$

Since the LOT is obtained from the even and odd value of $k$, we focus on the discussion of the dual generation for the CLT only. Define an Auxiliary Complex Lapped Transform (ACLT) of $2 N$ samples $\left[x\left(t-N+\frac{1}{2}\right), x\left(t-N+\frac{3}{2}\right), \cdots, x\left(x+N-\frac{1}{2}\right)\right]$ as

$$
\begin{aligned}
& X_{c l t}(k, t)= \frac{1}{\sqrt{N}} \sum_{n=t-\left(N-\frac{1}{2}\right)}^{t+\left(N-\frac{1}{2}\right)} x(n) \\
& \cdot \exp \left\{-j \frac{(2 k+1)(n-t) \pi}{2 N}\right\} \sin \frac{(n-t) \pi}{2 N}, \\
& k=0,1, \cdots, N-1 .
\end{aligned}
$$

Then, the CLT and ACLT can be dually generated from (2) and (3) with the corresponding coefficients listed in Table II. All the transforms mentioned above can be realized by a lattice structure as shown in Fig. 1. This lattice structure is a modified normal form digital filter. Table II lists the coefficients in the unified lattice structure for different transforms. Here $\theta_{k}$ associated with the LOT/CLT equals $\frac{(2 k+1) \pi}{4 N}$.

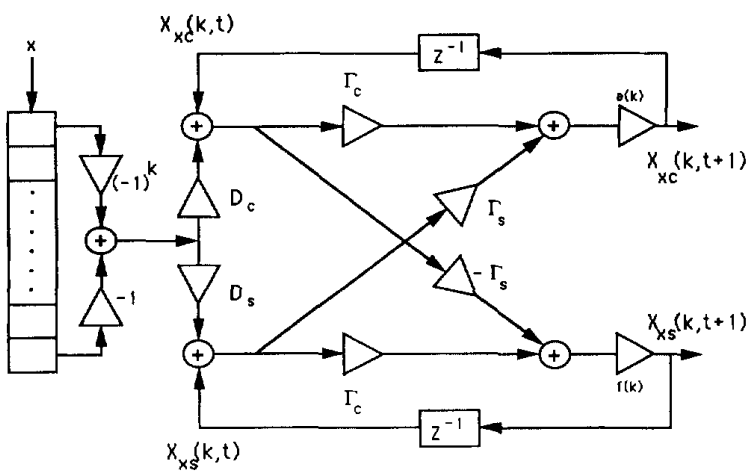

Fig. I. The universal lattice module.

\section{Optimal Time-Recursive ArChitectures}

\section{III.I. Transfer Function Approach}

Input data arrive serially in most real-time signal processing applications. If we can view the transform operation as a linear shift invariant (LSI) system which transforms the input sequence of samples into their transform coefficients, then it is similar to a filtering operation. The general approach to tackle a digital filter problem is to look at its transfer function. The transfer functions of the DXT can be derived using several approaches. We will derive them from the unified time-recursive lattice structures as shown in Fig. 1. The time difference equations ${ }^{1}$ for the dually generated pairs are

$y_{x c, k}(t)=e(k)\left\{\Gamma_{c}\left[D_{c} \tilde{x}(t)+y_{x c, k}(t-1)\right]\right.$

$$
\left.+\Gamma_{c}\left[D_{s} \tilde{x}(t)+y_{x s, k}(t-1)\right]\right\}
$$

and

$$
\begin{aligned}
y_{x s, k}(t)=f(k)\left\{\Gamma_{c}\left[D_{s} \tilde{x}(t)+y_{x s, k}(t-1)\right]\right. \\
\left.-\Gamma_{s}\left[D_{c} \tilde{x}(t)+y_{x c, k}(t-1)\right]\right\},
\end{aligned}
$$

where

$$
\tilde{x}(t)=(-1)^{k} x(t+N)-x(t),
$$

and $y_{x c, k}(t)$ and $y_{x s, k}(t)$ corresponds to $X_{x c}(k, t)$ and $X_{x s}(k, t)$ in (2) and (3). The $z$ transform deduced from the above difference equations are

$$
\begin{aligned}
Y_{x c, k}(z)=e(k)\{ & \left(D_{c} \Gamma_{c}+D_{s} \Gamma_{s}\right) \tilde{X}(z) \\
& \left.+\Gamma_{c} Y_{x c, k}(z) z^{-1}+\Gamma_{s} Y_{x s, k}(z) z^{-1}\right\}
\end{aligned}
$$

' The time index $t$ is an integer parameter. 
TABLE III

Coefficients of the Universal IIR FILter Structure for the DXT

\begin{tabular}{|c|c|c|c|c|c|c|}
\hline & $k$ & $n$ & $D 1$ & $D 2$ & $N 1$ & $N 2$ \\
\hline DCT & $k$ & $N$ & $2 \cos (\pi k / N)$ & $\begin{array}{l}1 \\
1\end{array}$ & $\begin{array}{l}C(k) \sqrt{\frac{2}{N}} \\
\cdot \cos (\pi k / 2 N)\end{array}$ & $\begin{array}{l}-C(k) \sqrt{\frac{2}{N}} \\
\cdot \cos (\pi k / 2 N)\end{array}$ \\
\hline DST & $k$ & $N$ & $2 \cos (\pi k / N)$ & 1 & $\begin{array}{l}-C(k) \sqrt{\frac{2}{N}} \\
\cdot \sin (\pi k / 2 N)\end{array}$ & $\begin{array}{l}-C(k) \sqrt{\frac{2}{N}} \\
\cdot \sin (\pi k / 2 N)\end{array}$ \\
\hline DHT & 0 & $N$ & $2 \cos (2 \pi k / N)$ & 1 & $\begin{array}{l}\sqrt{\frac{1}{N}}[\cos (2 \pi k / N)- \\
\sin (2 \pi k / N)]\end{array}$ & $\sqrt{\frac{1}{N}}$ \\
\hline DFT & 0 & $N$ & $2 \cos (2 \pi k / N)$ & l & $\begin{array}{l}\sqrt{\frac{1}{N}}[\cos (2 \pi k / N)+ \\
j \sin (2 \pi k / N)]\end{array}$ & $\sqrt{\frac{1}{N}}$ \\
\hline CLT & 0 & $2 N$ & $\begin{array}{l}\exp \left(j 2 \theta_{k}\right) \\
\cdot 2 \cos (\pi / 2 N) \\
\end{array}$ & $\exp \left(j 4 \theta_{k}\right)$ & $\begin{array}{l}\sin (\pi / 4 N)^{2} \\
\exp \left(j \theta_{k}\right)\end{array}$ & $\begin{array}{l}(-1)^{k} \sin (\pi / 4 N) \\
\cos (\pi / 4 N) \exp \left(j 4 \theta_{k}\right)\end{array}$ \\
\hline
\end{tabular}

and

$$
\begin{aligned}
Y_{x s, k}(z)=f(k) & \left\{\left(D_{s} \Gamma_{c}-D_{c} \Gamma_{s}\right) \tilde{X}(z)\right. \\
& \left.+\Gamma_{c} Y_{x s, k}(z) z^{-1}-\Gamma_{s} Y_{x c, k}(z) z^{-1}\right\} .
\end{aligned}
$$

$Y_{x s, k}(z)$ can be expressed in terms of $Y_{x c, k}(z)$ and $\tilde{X}(z)$ as

$$
Y_{x s, k}(z)=\frac{f(k)\left\{\left(D_{s} \Gamma_{c}-D_{c} \Gamma_{s}\right) \tilde{X}(z)-\Gamma_{s} Y_{x c . k}(z) z^{-1}\right\}}{1-f(k) \Gamma_{c} z^{-1}}
$$

it follows that the transfer function for $\frac{Y_{x c k}(z)}{\bar{X}(z)}$ is given by

$$
\begin{aligned}
& H_{x c, k}(z) \\
& =\frac{\left((-1)^{k}-z^{-N}\right)\left(e(k)\left[D_{c} \Gamma_{c}+D_{s} \Gamma_{s}\right]-e(k) f(k) D_{c} z^{-1}\right)}{1-(e(k)+f(k)) \Gamma_{c} z^{-1}+e(k) f(k) z^{-2}} .
\end{aligned}
$$

Similarly, the transfer function for $\frac{Y_{x s . k}(Z)}{X(z)}$ is given by

$$
\begin{aligned}
& H_{x s, k}(z) \\
& =\frac{\left((-1)^{k}-z^{-N}\right)\left(f(k)\left[D_{s} \Gamma_{c}-D_{c} \Gamma_{s}\right]-e(k) f(k) D_{s} z^{-1}\right)}{1-(e(k)+f(k)) \Gamma_{c} z^{-1}+e(k) f(k) z^{-2}} .
\end{aligned}
$$

From Table II and the transfer functions derived above, the transfer functions of different discrete sinusoidal transforms are given by

$$
\begin{gathered}
H_{c}(z)=\sqrt{\frac{2}{N}} C(k)\left((-1)^{k}-z^{-N}\right)\left(\cos \frac{\pi k}{2 N}\right) \\
\cdot \frac{\left(1-z^{-1}\right)}{\left(1-2\left(\cos \frac{\pi k}{N}\right) z^{-1}+z^{-2}\right)}, \\
H_{s}(z)=-\sqrt{\frac{2}{N}} C(k)\left((-1)^{k}-z^{-N}\right)\left(\sin \frac{\pi k}{2 N}\right) \\
\cdot \frac{\left(1+z^{-1}\right)}{\left(1-2\left(\cos \frac{\pi k}{N}\right) z^{-1}+z^{-2}\right)}, \\
H_{h}(z)=\frac{1}{\sqrt{N}}\left(1-z^{-N}\right)\left(\frac{\cos \frac{2 \pi k}{N}-\sin \frac{2 \pi k}{N}-z^{-1}}{1-2\left(\cos \frac{2 \pi k}{N}\right) z^{-1}+z^{-2}}\right), \\
H_{f}(z)=\frac{1}{\sqrt{N}}\left(1-z^{-N}\right)\left(\frac{\cos \frac{2 \pi k}{N}+j \sin \frac{2 \pi k}{N}-z^{-1}}{1-2\left(\cos \frac{2 \pi k}{N}\right) z^{-1}+z^{-2}}\right) .
\end{gathered}
$$

Because the size of the input data is $2 N$ in the CLT, the updating vector is $1-z^{2 N}$ instead of $1-z^{N}$. The transfer function is obtained by substituting the corresponding coefficients in Table II to (11), resulting in

$$
\begin{gathered}
H_{c l t}(z)=\left(1-z^{-2 N}\right) \frac{1}{\sqrt{N}} j(-1)^{k+1} \\
\cdot \frac{\left(\sin \frac{\pi}{4 N}\right) e^{j \theta}\left(1+e^{j 2 \theta} z^{-1}\right)}{1-e^{j 2 \theta} 2\left(\cos \frac{\pi}{2 N}\right) z^{-1}+e^{j 4 \theta} z^{-2}}, \\
\theta=\frac{(2 k+1) \pi}{4 N} .
\end{gathered}
$$

It follows that for the LOT,

$$
\begin{aligned}
& H_{\text {lote }}(z)=\text { evenpartof }\left\{H_{c l t}\right\} \\
& H_{\text {loto }}(z)=\text { oddpartof }\left\{H_{c l t}\right\} .
\end{aligned}
$$

We know from (1) that the transfer functions of these transforms are of finite impulse response. Hence, the poles in the denominator will be cancelled by the zeros of $\left((-1)^{k}-z^{-N}\right)$ in the nominator. We observe that when the updating vector $\left(1-z^{-N}\right)$ is factored out, the basic structure of all the transforms is composed of a FIR and an IIR filter with a second order denominator and a first order numerator, i.e. we are using an IIR filter to realize a FIR filter. This realization can greatly reduce the hardware complexity compared with the implementation consisting of FIR structures.

\section{III.2. The Unified IIR Filter Architectures}

From the transfer functions derived above, we observe that the DXT can be realized using a single universal filter module consisting of a shift register array and a second order IIR filter. This structure is depicted in Fig. 2. The coefficients of the universal IIR module for different transforms are listed in Table III.

We note from (19) and (20) that the DCT and the DST share the same denominator and can be simultaneously generated using an IIR filter structure with three multipliers as depicted in Fig. 3. Compared with the lattice structure for the DCT and DST [1], the IIR realization requires only half as many multipliers. The difference is that the IIR structure implements the denominator of the transfer function in the direct form, while the lattice structure implements the poles in the normal 


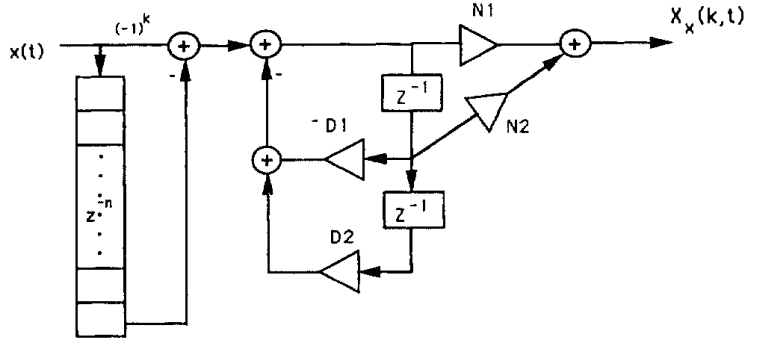

Fig. 2. The universal IIR filter module.

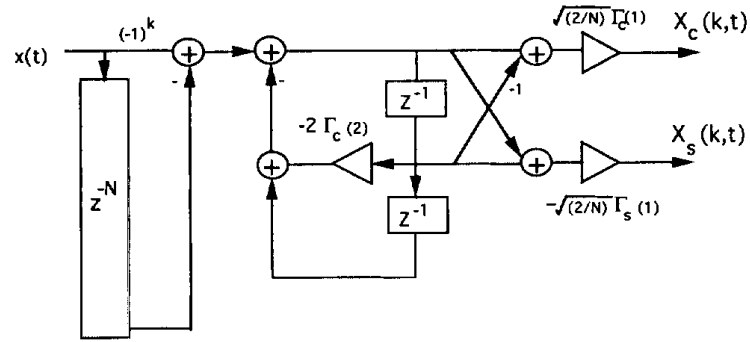

Fig. 3. The IIR filter structure for the DCT and DST.

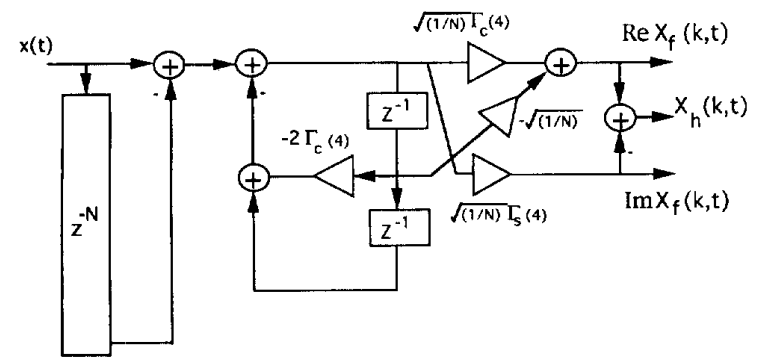

Fig. 4. The IIR filter structure for the DHT and DFT.

form. From (21) and (22), we also observe that a single unified filter structure can be used to generate both the DHT and the DFT. This structure is depicted in Fig. 4.

The transfer function derived in (23) is in complex form. We will show in the following how to realize the CLT using real operations. The definition of the CLT in (9) can be rewritten as

$$
\begin{aligned}
& X_{c l t}(k)=(-1)^{k} j \frac{1}{\sqrt{N}} \sum_{n=0}^{2 N-1} x(n) \\
& \cdot \exp \left\{-j \frac{(2 k+1)(2 n+1) \pi}{4 N}\right\} \sin \frac{(2 n+1) \pi}{4 N}, \\
& k=0,1, \cdots, N-1
\end{aligned}
$$

If we define another transform with basis functions only length $N$,

$$
\begin{array}{r}
t_{n k}=\frac{1}{N} \exp \frac{j(2 n+1) k \pi}{2 N}=\frac{1}{N}\left\{D C T_{n k}-j \cdot D S T_{n k}\right\} \\
n, k=0,1, \cdots, N-1
\end{array}
$$

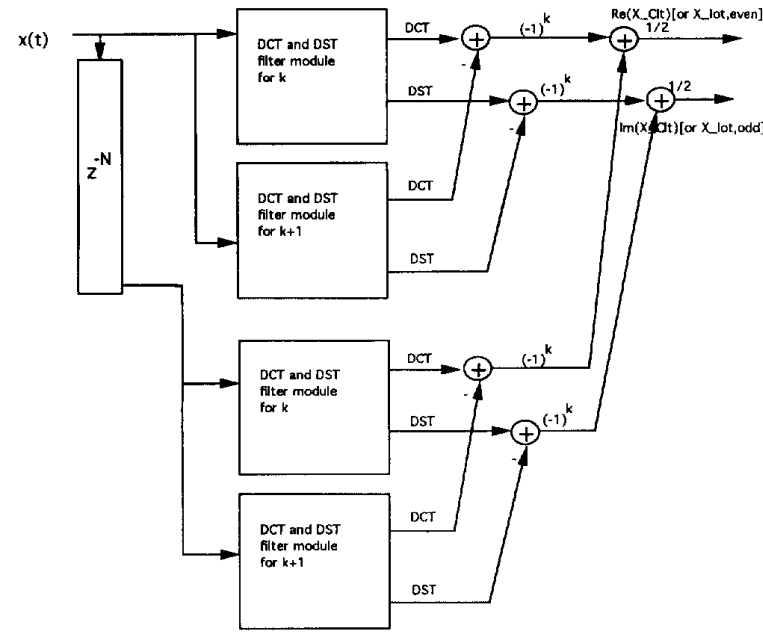

Fig. 5. The IIR filter structure for real operation of the LOT and CLT.

TABLE IV

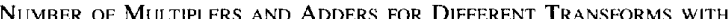
IIR Filter Realizations (Here * Denotes Complex Operations)

\begin{tabular}{lll}
\hline Transformers & multipliers & adders \\
\hline DCT & $2 N-2$ & $3 N+2$ \\
DST & $2 N-2$ & $3 N+2$ \\
DHT & $2 N$ & $3 N+1$ \\
DFT & $3 N-2$ & $3 N+1$ \\
LOT $^{*}$ & $4 N$ & $4 N$ \\
CLT $^{*}$ & $4 N$ & $4 N$ \\
DCT and DST & $3 N-3$ & $4 N+2$ \\
DHT and DFT & $3 N-2$ & $4 N+1$ \\
\hline
\end{tabular}

then the CLT can be expressed in the form of [13]

$$
\begin{aligned}
X_{c l t}(k)=\frac{1}{2}(-1)^{k} & \sum_{n=0}^{N-1} x(n)\left[t_{n k}-t_{n(k+1)}\right] \\
& +\frac{1}{2}(-1)^{k} \sum_{n=N}^{2 N-1} x(n)\left[t_{n k}+t_{n(k+1)}\right],
\end{aligned}
$$

This leads to the CLT architecture as shown in Fig. 5, in which the $t_{m n}$ are generated by using the DCT and DST dual generating circuit as depicted in Fig. 3. The number of multipliers and adders required for these IIR filter structures are summarized in Table IV.

The architecture to generate 1-D DXT is depicted in Fig. 6. This parallel structure consists of a shift register array of size $N$, two adders, and $N$ IIR filter modules. Two sets of inputs $x(t+N)-x(t)$ and $-x(t+N)-x(t)$ are generated for the even and odd filter modules respectively. When a new datum $x(t)$ arrives, a new set of transform coefficients are obtained in $O(1)$ time, i.e., the throughput rate is $O(1)$.

\section{ARCHITECTURES FOR INVERSE TRANSFORMS}

Inverse transforms are important in retrieving original information in digital communication systems. The inverse DHT 


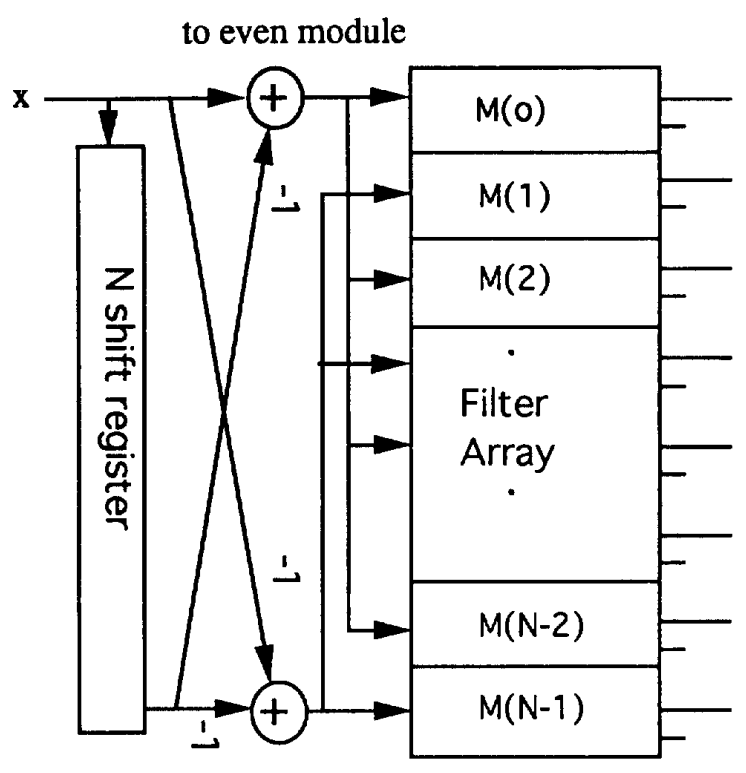

to odd module

Fig. 6. The parallel IIR filter structure for 1-D DXT.

and DFT are given by

$$
\begin{gathered}
x_{h}(n, t)=\frac{1}{\sqrt{N}} \sum_{k=t}^{t+N-1} X_{h}(k) \operatorname{cas}\left(2 n \frac{\pi(k-t)}{N}\right) \\
n=0,1, \cdots, N-1 . \\
x_{f}(n, t)=\frac{1}{\sqrt{N}} \sum_{k=t}^{t+N-1} X_{f}(k) \exp \left\{j 2 n \frac{\pi(k-t)}{N}\right\}, \\
n=0,1, \cdots, N-1 .
\end{gathered}
$$

We observe that the transfer function of the inverse DHT (IDHT) is exactly the same as its forward transform. The transfer function of the inverse DFT (IDFT) is given by

$$
\begin{aligned}
H_{f}(z)=\frac{1}{\sqrt{N}}\left(1-z^{-N}\right) & \\
& \cdot\left(\frac{\cos \frac{2 \pi k}{N}-j \sin \frac{2 \pi k}{N}-z^{-1}}{1-2 \cos \frac{2 \pi k}{N} z^{-1}+z^{-2}}\right),
\end{aligned}
$$

which is the same as (22) except that the imaginary part is negated. Therefore, the IDHT and IDFT can be realized by using the same architecture as those depicted in Fig. 4 except that we have to add an inverter at the output of the $\operatorname{Im} X_{f}(k, t)$.

The inverse DCT and DST (IDCT and IDST) are defined as follows:

$$
\begin{aligned}
x_{c}(n, t)= & \sqrt{\frac{2}{N}} \sum_{k=t}^{t+N-1} C(k-t) X_{c}(k) \\
& \cdot \cos \left[\left(n+\frac{1}{2}\right) \frac{(k-t) \pi}{N}\right], \\
& n=0,1, \cdots, N-1 .
\end{aligned}
$$

$$
\begin{aligned}
x_{s}(n, t)= & \sqrt{\frac{2}{N}} \sum_{k=t+1}^{t+N} C(k-t) X_{s}(k) \\
& \cdot \sin \left[\left(n+\frac{1}{2}\right) \frac{(k-t) \pi}{N}\right], \\
n & =0,1, \cdots, N-1 .
\end{aligned}
$$

Because $C(k)$ is inside the transform, the architectures require some modification. Since $C(k)=1$ except for $k=0$ or $k=N$, we can rewrite (32) as

$$
\begin{aligned}
x_{c}(n, t)=\sqrt{\frac{2}{N}} \sum_{k=t}^{t+N-1} X(k) & \cos \left[\left(n+\frac{1}{2}\right) \frac{(k-t) \pi}{N}\right] \\
+ & \sqrt{\frac{2}{N}}\left(\sqrt{\frac{1}{2}}-1\right) X(t) .
\end{aligned}
$$

The transfer function of IDCT is

$$
\begin{array}{r}
H_{i c}(z)=\sqrt{\frac{2}{N}} \frac{z^{-N-1}-\cos \theta z^{-N}+(-1)^{n} \sin \theta}{1-2 \cos \theta z^{-1}+z^{-2}}, \\
+\sqrt{\frac{2}{N}}\left(\sqrt{\frac{1}{2}}-1\right) z^{-(N-1)}
\end{array}
$$

where $\theta=\frac{\pi(n+.5)}{N}$. If we perform the block transform instead of sliding window transform, then the $z^{-N-1}$ and $z^{-N}$ components in the numerator can be eliminated because of the reset operation. In Fig. 7, we show the optimal unified IIR implementation of the inverse DCT module under block transform. The number of multipliers required for the inverse DCT is $2 N-1$. The additional branch of multiplier is shared by the $N$ IIR array with a delay of $N-1$ cycles. The difference in the direct and inverse transform formula can be rectified by adding one additional branch of multipliers to a whole parallel IIR structure and changing the multiplication coefficients. Similarly, the IDST can be rewritten as

$$
\begin{aligned}
x_{s}(n, t)=\sum_{k=t}^{t+N-1} X(k) \sin \left[\frac{\pi(2 n+1)(k-t)}{2 N}\right] \\
+\sqrt{\frac{2}{N}}\left(\sqrt{\frac{1}{2}}-1\right) X(t+N-1)
\end{aligned}
$$

whose transfer function is

$$
\begin{array}{r}
H_{i s}(z)=\sqrt{\frac{2}{N}} \frac{\sin \theta z^{-N}-(-1)^{n} z^{-1}+(-1)^{n} \cos \theta}{1-2 \cos \theta z^{-1}+z^{-2}} \\
+\sqrt{\frac{2}{N}}\left(\sqrt{\frac{1}{2}}-1\right)
\end{array}
$$

The architecture for the block transform of the IDST is shown in Fig. 8.

The Inverse Complex Lapped Transform (ICLT) [13] of samples $[X(t), X(t+1), \cdots, X(t+N-1), X(t+$ $N), \cdots, X(t+2 N-1)]$ is defined as

$$
\begin{aligned}
x_{c l t}(k, t) & \frac{1}{2 \sqrt{N}} \sum_{k=t}^{t+N-1}\left[X(k)+X(k+1)+(-1)^{k}(X(k+N)\right. \\
& +X(k+N+1))] \exp \left\{j \frac{(2 n+1)(k-t) \pi}{2 N}\right\} .
\end{aligned}
$$


TABLE V

Corresponding Coefficients in the Recurrence Formula for Different DXT

\begin{tabular}{|c|c|c|c|c|c|c|c|}
\hline & $k$ & $c$ & $\lambda$ & $P_{0}$ & $P_{-1}$ & $P_{N-1}$ & $P_{N}$ \\
\hline$\overline{\mathrm{DCT}}$ & $2 \cos (\pi k / N)$ & 0 & 1 & $\cos (\pi k / 2 N)$ & $P_{0}$ & $(-1)^{k} P_{0}$ & $P_{0}$ \\
\hline DST & $2 \cos (\pi k / N)$ & 0 & 1 & $\sin (\pi k / 2 N)$ & $-P_{0}$ & $-(-1)^{k} P_{0}$ & $P_{0}$ \\
\hline DHT & $2 \cos (2 \pi k / N)$ & 0 & 1 & 1 & $\begin{array}{l}\cos (2 \pi k / 2) \\
\sin (2 \pi k / N)\end{array}$ & $P_{-1}$ & 1 \\
\hline DFT & $2 \cos (2 \pi k / N)$ & 0 & 1 & 1 & $\begin{array}{l}\cos (2 \pi k / 2) \\
-j \sin (2 \pi k / N)\end{array}$ & $P_{-1}$ & 1 \\
\hline CLT & $\begin{array}{l}\exp \left(-j 2 \theta_{k}\right) \\
2 \cos (\pi / 2 N)\end{array}$ & 0 & $\exp \left(-j 4 \theta_{k}\right)$ & 1 & $\begin{array}{l}\exp \left(j 2 \theta_{k}\right) \\
\cdot \cos (\pi / 2 N)\end{array}$ & $\begin{array}{l}(-1)^{k} \\
-\sin (\pi / 2 N) \\
j \exp j 2 \theta_{k}\end{array}$ & $(-1)^{k} j$ \\
\hline
\end{tabular}

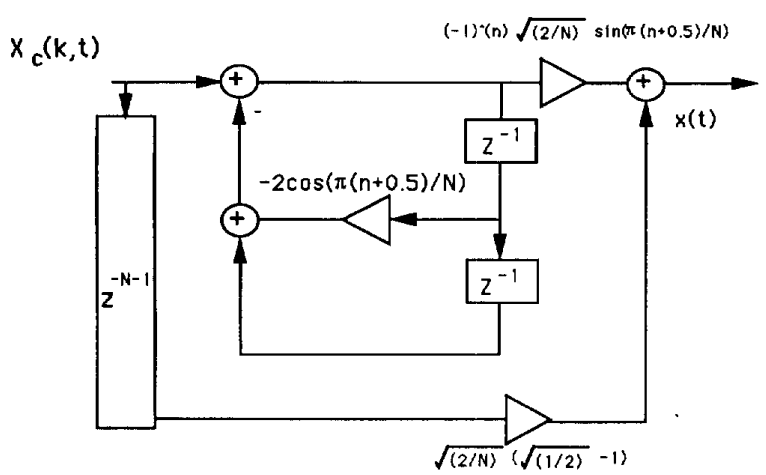

Fig. 7. The IIR filter structure for the IDCT.

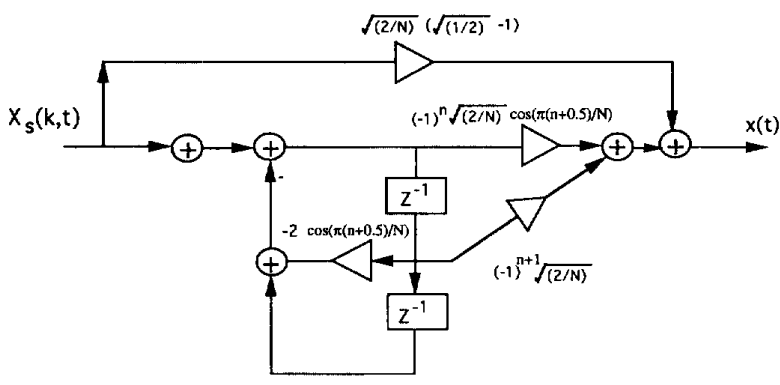

Fig. 8. The IIR filter structure for the IDST.

To compute the ICLT, the $2 N$ inputs are combined first into length of $N$, then the IDCT and IDST of the $N$-point vector are calculated individually. The ICLT is obtained by summing the result of the IDCT and that of IDST multiplied by $j$. The architecture of the IDCLT is depicted in Fig. 9.

\section{THEORETICAL BASIS}

The basis functions of all the discrete sinusoidal transforms mentioned above corresponds to a set of orthogonal polynomials [15]. One of the important characteristics of orthogonal polynomials is that any three consecutive polynomials $P_{n}(k)$ are related by the Fundamental Recurrence Formula [16] given by

$$
P_{n}(k)=\left(k-c_{n}\right) P_{n-1}(k)-\lambda_{n} P_{n-2}(k) .
$$

The discrete transforms discussed in the previous section satisfy a simpler version of the recurrence relation. More

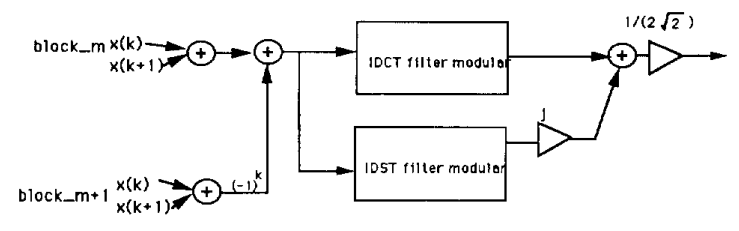

Fig. 9. The IIR filter structure for the IDCLT.

precisely, the parameters $c_{n}$ and $\lambda_{n}$ are independent of $n$ and the basis function $P_{n}(k)$ is periodic in $n$ and $k$ of period $N$. In these cases, the Fundamental Recurrence Formula can be rewritten as

$$
\begin{aligned}
P_{n}(k) & =(k-c) P_{n-1}(k)-\lambda P_{n-2}(k) \\
& n=0,1, \cdots, N-1, \quad k=0,1,2, \cdots, N-1 .
\end{aligned}
$$

For different discrete sinusoidal transforms, the corresponding parameters $k, c, \lambda$ in the Fundamental Recurrence Formula are stated in Table V.

Lemma 1: For all discrete transforms whose basis functions satisfy the Fundamental Recurrence Formula (42), the $z$ transform of the basis functions $\left\{P_{n}(k)\right\}$ can be expressed as a rational function with a second order denominator that is the characteristic equation of the Fundamental Recurrence Formula.

Proof: Since any $P_{n}(k)$ depends only on the previous two terms, the first two polynomial terms, $P_{-1}(k)$ and $P_{-2}(k)$, uniquely specify the entire set of basis functions.

Apply $z$ transform on index $n$ to both sides of (42),

$$
\begin{aligned}
P(z, k) & \\
= & \sum_{n=0}^{N-1} z^{-n} P_{n}(k) \\
= & \sum_{n=0}^{N-1}\left\{(k-c) z^{-n} P_{n-1}(k)-\lambda z^{-n} P_{n-2}(k)\right\} \\
= & (k-c)\left[P_{-1}(k)+z^{-1} \sum_{n=0}^{N-1} z^{-n} P_{n}(k)-z^{-N} P_{N-1}(k)\right] \\
& -\lambda\left[P_{-2}(k)+z^{-1} P_{-1}(k)+z^{-2} \sum_{n=0}^{N-1} z^{-n} P_{n}(k)\right. \\
& \left.-z^{-N} P_{N-2}(k)-z^{-(N+1)} P_{N-1}(k)\right]
\end{aligned}
$$




$$
\begin{aligned}
P(z, k) & =\frac{z^{-(N-1)} \lambda P_{N-1}(k)-P_{N}(k) z^{-(N-2)}-\lambda P_{-1}(k)+P_{0}(k) z^{2}}{\lambda-(k-c) z+z^{2}} \\
& =\frac{z^{2}\left(P_{0}(k)-P_{N}(k) z^{-N}\right)-\lambda z\left(P_{-1}(k)-P_{N-1}(k) z^{-N}\right)}{\lambda-(k-c) z+z^{2}} .
\end{aligned}
$$

$$
\begin{aligned}
= & (k-c) z^{-1} P(z, k)-\lambda z^{-2} P(z, k) \\
& +\left[(k-c) P_{-1}(k)-\lambda P_{-2}(k)\right]-\lambda z^{-1} P_{-1}(k) \\
& -z^{-N}\left[(k-c) P_{N-1}(k)-\lambda P_{N-2}(k)\right] \\
& +\lambda z^{-(N+1)} P_{N-1}(k) \quad \\
& \quad k=1,2, \cdots, N-1 .
\end{aligned}
$$

Factoring out $P(z, k)$, we obtain (see (44) at top of page) Because of the second order recurrence relation, the denominators of the $z$-transform of the basis functions are second-order polynomials in $z$.

The characteristic equation of the Fundamental Recurrence Formula (42) is obtained by solving the homogeneous solutions of the difference equation (42). The homogeneous equation is given by

$$
P(z, k)=(k-c) P(z, k) z^{-1}-\lambda P(z, k) z^{-2} .
$$

Combining both sides of the equation, we have

$$
P(z, k) z^{-2}\left(\lambda-(k-c) z+z^{2}\right)=0 .
$$

Since $P(z, k)$ does not equal to zero, we have that $(\lambda-(k-$ c) $\left.z+z^{2}\right)$ equals to zero and hence the characteristics equation is $\left(\lambda-(k-c) z+z^{2}\right)$, which is the denominator.

The transfer function of the discrete transforms (DXT) is derived from (1), that can be rewritten as

$$
X(k, t)=C(k) \sum_{n=0}^{N-1} x(n+t) P_{n}(k), t=0,1,2, \ldots
$$

Performing the $z$-transform on the index $t$ on both sides of the above equation, we have

$$
\begin{aligned}
H_{\mathrm{X}}(z) & =C(k) z^{-(N-1)} \sum_{n=0}^{N-1} z^{n} P_{n}(k) \\
& =C(k) z^{-(N-1)} P\left(z^{-1}, k\right)
\end{aligned}
$$

which is the $z$-transform of the basis orthogonal polynomials with index $z$ replaced by $z^{-1}$ and multiplied by $C(k) z^{-(N-1)}$. That is, the transfer function of the discrete transform can also be expressed as a rational function with a second order denominator

$$
\begin{aligned}
& H_{x}(z)=C(k) \\
& \frac{\left(\lambda P_{N-1}(k)-P_{N}(k) z^{-1}-\lambda P_{-1}(k) z^{-N}+P_{0}(k) z^{-(N+1)}\right)}{\left(\lambda-(k-c) z^{-1}+z^{-2}\right)} .
\end{aligned}
$$

Here we illustrate another way to derive the transfer function of the discrete sinusoidal transforms. Substituting the coefficients listed in Table $\mathrm{V}$ to (49), we obtain the transfer functions derived in Section III.1.
Lemma 2: To compute the discrete sinusoidal transforms time recursively, we have to factor out the updating component $\left(1-z^{-N}\right)$ or $\left(1+z^{-N}\right)$ in the filter realization. There exists an updating component $\left(1+z^{-N}\right)$ or $\left(1-z^{-N}\right)$ in the nominator of the transfer function of the discrete sinusoidal transform, if and only if the boundary conditions of the basis function satisfy $P_{0}= \pm P_{N}$ and $P_{-1}= \pm P_{N-1}$.

Proof: If the updating vector can be realized by $(1+$ $\left.z^{-N}\right)$ or $\left(1-z^{-N}\right)$, then the nominator of (49) must contain the factor $\left(1+z^{-N}\right)$ or $\left(1-z^{-N}\right)$. That is, the nominator can be expressed as

$$
\begin{aligned}
\lambda P_{N-1}(k)-P_{N}(k) z^{-1}-\lambda P_{-1}(k) z^{-N}+P_{0}(k) z^{-(N+1)} \\
=\left(1 \pm z^{-N}\right)\left(a+b z^{-1}\right)
\end{aligned}
$$

since it is a $(-N-1)$ degree polynomial. Expand the right side of the above equation, we have

$$
\begin{aligned}
\lambda P_{N-1}(k)-P_{N}(k) z^{-1} & -\lambda P_{-1}(k) z^{-N}+P_{0}(k) z^{-(N+1)} \\
& =a+b z^{-1} \pm a z^{-N} \pm b z^{-N-1}
\end{aligned}
$$

it follows that

$$
\begin{aligned}
& a=\mp \lambda P_{-1}(k)=\lambda P_{N-1}(k) \\
& b= \pm P_{0}(k)=-P_{N}(k),
\end{aligned}
$$

and

$$
\begin{gathered}
P_{0}(k)= \pm P_{N}(k) \\
P_{-1}(k)=\mp P_{N-1}(k) .
\end{gathered}
$$

This proves the necessary condition. If $P_{0}= \pm P_{N}$ and $P_{-1}= \pm P_{N-1}$, then the nominator in (49) becomes

$$
\begin{aligned}
& \lambda P_{N-1}(k)-P_{N}(k) z^{-1}-\lambda P_{-1}(k) z^{-N}+P_{0}(k) z^{-(N+1)} \\
& \quad=\mp \lambda P_{-1}(k) \pm P_{0}(k) z^{-1}-\lambda P_{-1}(k) z^{-N} \\
& \quad+P_{0}(k) z^{-(N+1)} \\
& \quad=\left(1 \pm z^{-N}\right)\left(\lambda P_{0}(k) z^{-1} \mp \lambda P_{-1}(k)\right)
\end{aligned}
$$

which means the nominator contains the factor $\left(1 \pm z^{-N}\right)$.

Lemma 3: All the transforms that satisfies Lemma 1 and Lemma 2 can be realized by an updating FIR filter with transfer function $\left(1-z^{-N}\right)$ or $\left(1+z^{-N}\right)$, and an IIR filter with second order denominator and first order nominator whose coefficients are dependent on $\lambda,(k-c), P_{0}$ and $P_{-1}$.

Proof: If Lemma 1 and 2 are satisfied, the transfer function can be expressed as

$$
H_{z}(z)=C(k) \frac{\left(1 \pm z^{-N}\right)\left(\lambda P_{N-1}-P_{N} z^{-1}\right)}{\left(\lambda-(k-c) z^{-1}+z^{-2}\right)} .
$$


Therefore, the transform can be realized by the filter structure as shown in Fig. 2. The coefficients are

$$
\begin{array}{ll}
D 1=(k-c) & D 2=\lambda \\
N 1=\lambda P_{N-1} & N 2=-P_{N} .
\end{array}
$$

Lemma 3 implies that if a transform can be computed timerecursively, a maximum of four multipliers required to realize the transform. Fig. 2 shows a good example of this case.

Lemma 4: For the discrete sinusoidal transforms, the roots of the characteristic equation belong to the set of the root of $\left(1 \pm z^{-N}\right)$.

Proof: Since the discrete sinusoidal transform is FIR in natural, the roots of the denominators should be cancelled by the zeros of the nominator. In general, the roots of the denominator are complex conjugate poles because of ( $k-$ $c)^{2}-4 \lambda<0$. Therefore, the poles should be cancelled by the zeros of the $\left(1 \pm z^{-N}\right)$, and the roots of the denominator

$$
\begin{aligned}
& z 1, z 2=\frac{(k-c) \pm \sqrt{(k-c)^{2}-4 \lambda}}{2 \lambda} \\
& \in\left\{\begin{array}{ll}
\exp \frac{j 2 \pi n}{N} & n=0,1,2, \cdots, N-1 \cdot z^{N}=1 \\
\exp \frac{j \pi(2 n+1)}{N} & n=0,1,2, \cdots, N-1 \cdot z^{N}=-1 .
\end{array}\right\}
\end{aligned}
$$

All the discrete sinusoidal transforms list in Table IV satisfies Lemmas 1 through 4 . Therefore, these transforms can be computed time recursively and can be realized by a FIR filter with transfer function $\left(1 \pm z^{-N}\right)$ and an IIR filter with second order polynomials. These facts support the results obtained in Section III and IV.

Lemma 5: If two transforms can be dually generated, then they share the same autoregressive model in their IIR filter structure.

Proof: The basis polynomial $p_{n}$ and $q_{n}$ of the dual generated transform pairs satisfy the following equations

$$
\begin{aligned}
& p_{n}=D_{x c} p_{n-1}+D_{x s} q_{n-1} \\
& q_{n}=D_{x c} q_{n-1}-D_{x s} p_{n-1} .
\end{aligned}
$$

Since $p_{n}$ and $q_{n}$ are dually generated and from (59), they have the same characteristic equation. That is

$$
I-A z^{-1}=0 \text {. }
$$

where

$$
A=\left[\begin{array}{cc}
D_{x c} & D_{x s} \\
-D_{x s} & D_{x c}
\end{array}\right]
$$

As shown in Lemma 1, the roots of the denominators are the roots of the characteristics equation. Since $p_{n}$ and $q_{n}$ have the same characteristic equation, they have the same denominator. Hence, both transform have identical poles, and as a result, the same autoregressive filter form

Example 1: The DCT and DST are dual generated transform pairs and share the same second order denominator.

As shown in [1], the DCT and DST satisfy

$$
\begin{aligned}
\cos \left[\frac{\pi(2(n+1)+1) k}{2 N}\right]= & \cos \left[\frac{\pi k}{N}\right] \cos \left[\frac{\pi(2 n+1) k}{2 N}\right] \\
& -\sin \left[\frac{\pi k}{N}\right] \sin \left[\frac{\pi(2 n+1) k}{2 N}\right]
\end{aligned}
$$

$$
\begin{aligned}
\sin \left[\frac{\pi(2(n+1)+1) k}{2 N}\right]= & \cos \left[\frac{\pi k}{N}\right] \sin \left[\frac{\pi(2 n+1) k}{2 N}\right] \\
& +\sin \left[\frac{\pi k}{N}\right] \cos \left[\frac{\pi(2 n+1) k}{2 N}\right],
\end{aligned}
$$

it follows that

$$
\begin{aligned}
& D_{x c}=\cos \left[\frac{\pi k}{N}\right], \\
& D_{x s}=-\sin \left[\frac{\pi k}{N}\right] .
\end{aligned}
$$

From (59), the poles are the root of the equation 1 $2 \cos [\pi k / N] z^{-1}+z^{-2}=0$, which is the same as the characteristic equation derived from the Lemma 1. This is why the DCT, DST and DFT, DHT share the same second order autoregressive structure. From Lemma 3, it is noted that a maximum of $4 N$ multipliers is required to realize the transform. Due to the fact that $\lambda=1$ and $P_{N}= \pm P_{N-1}$ for the case of the DCT and DST, we can see that $2 N$ multipliers for the DCT and DST is minimum for this realization. Based on Lemma 5, we can combine the denominator together for the dual generation of DCT and DST. This gives an average $1.5 \mathrm{~N}$ multipliers to realize the DCT or DST. We believe that this is the best we can achieve for real-time computation.

\section{UNIFIED TIME-Recursive Based}

\section{Multi-Dimensional Discrete SinUSOIDAL TRANSForms}

Multi-dimensional transforms provide powerful tools for multi-dimensional signal processing. Some of the important applications are in the areas of signal reconstruction, speech processing, spectrum analysis, tomography, image processing, and computer vision. Specifically, in multispectral imaging, interframe video imaging, and computer tomography, we have to work with three or (higher) dimensional data. It is difficult to generalize the existing fast 1-D algorithms to 3-D or higher dimensional transforms. However, our timerecursive concept can be easily extended to multi-dimensional transforms resulting in architectures that are simple, modular, and hence suitable for VLSI implementation. Since the 3-D DCT is very useful in processing interframe video imaging data, we first describe the filter architecture for the 3-D DCT, and then generalize it to any multi-dimensional discrete sinusoidal transform.

\section{VI.1. Time-Recursive Structures for 3-D DCT}

The basic concept of time-recursive computation is to compute the new transform at time $(t+1)$ based on the transform at time $t$. The operations can be divided into two parts, one consists of computing the difference of the input data between time $t$ and $(t+1)$ and the other consists of performing the recursive updating. Looking at the basic architecture of computing 1-D DXT as shown in Fig. 2, the basic structure consists of three components: shift registers, adders, and IIR arrays. The shift register is used to store the input data from $x(t)$ to $x(t+N)$; adders are used to compute the difference between data $x(t)$ and $x(t+N)$ and the IIR arrays are used 
to perform the computation recursively. We will show that the $d$-D DXT can be computed by using $d$ blocks consisting of shift registers, adders, and filter arrays, each performing the time-recursive computation along a dimension.

For 1-D time-recursive DXT, the input data window is moved one sample at a time. That is, the input data vector at time $t$ is given by the vector $[x(t), \cdots, x(t+N-1)]$, and at time $(t+1)$ the input data consists of the vector $[x(t+1), \cdots, x(t+N)]$. The time-recursive relation for the 2-D transforms is based on updating the input data row by row [17]. A 2-D DCT for HDTV application based on the lattice structure as considered in [17]. Assuming a 3-D input data is updated frame by frame in the third axis $n_{3}$ the range of the input data $x\left(n_{1}, n_{2}, n_{3}\right)$ is $\left\{n_{1}=0, \cdots, N-1 ; n_{2}=\right.$ $\left.0, \cdots, N-1 ; n_{3}=0,1,2, \ldots\right\}$, we call the input data frame $x\left(n_{1}, n_{2}, t\right)$ for a specific index $t$ as the $t$ th frame input data. The 3-D DCT of the $t$ th frame input data is defined as

$$
\begin{aligned}
& X_{c^{3}}\left(k_{1}, k_{2}, k_{3}, t\right) \\
& =C\left(k_{1}\right) C\left(k_{2}\right) C\left(k_{3}\right) \\
& \quad \cdot \sum_{n_{1}=0}^{N-1} \sum_{n_{2}=0}^{N-1} \sum_{n_{3}=t}^{t+N-1} x\left(n_{1}, n_{2}, n_{3}\right) \\
& \cdot \cos \left[\frac{\pi\left(2 n_{1}+1\right) k_{1}}{2 N}\right] \\
& \cdot \cos \left[\frac{\pi\left(2 n_{2}+1\right) k_{2}}{2 N}\right] \\
& \cdot \cos \left[\frac{\pi\left[2\left(n_{3}-t\right)+1\right] k_{3}}{2 N}\right] .
\end{aligned}
$$

By introducing another 3-D transform $X_{c^{2} s}\left(k_{1}, k_{2}, k_{3}, t\right)$ defined as

$$
\begin{aligned}
& X_{c^{2} s}\left(k_{1}, k_{2}, k_{3}, t\right) \\
& =C\left(k_{1}\right) C\left(k_{2}\right) C\left(k_{3}\right) \\
& \sum_{n_{1}=0}^{N-1} \sum_{n_{2}=0}^{N-1} \sum_{n_{3}=t}^{t+N-1} \cdot x\left(n_{1}, n_{2}, n_{3}\right) \cos \left[\frac{\pi\left(2 n_{1}+1\right) k_{1}}{2 N}\right] \\
& \cdot \cos \left[\frac{\pi\left(2 n_{2}+1\right) k_{2}}{2 N}\right] \\
& \quad \sin \left[\frac{\pi\left[2\left(n_{3}-t\right)+1\right] k_{3}}{2 N}\right] .
\end{aligned}
$$

By following the time-recursive approach, we can show that the 2-D DCT of each frame can be computed first and store it in a shift register array of size $(N+1) \times N^{2}$. The difference between the 2-D DCT of the $t$ th frame and $(t+N)$ th frame is then computed. The 3-D DCT can be generated by feeding the 2-D DCT of the updating vector into a lattice module as shown in Fig. 10. The size of the shift register in the lattice module is $N^{2}$ because for a specific $k_{3}$ there are $N^{2}$ values ( $k_{1}=0, \cdots, N-1 ; k_{2}=0, \cdots, N-1$ ) to be updated. A similar updating relation exists for the 2-D DCT and the 1-D DCT [17]. The number of shift registers in the lattice module for 2-D and 1-D DCT are $N$ and 1 respectively. Therefore, the time-recursive 3-D DCT lattice structure consists of three lattice arrays which are used to produce the 1-D, 2-D and 3-D

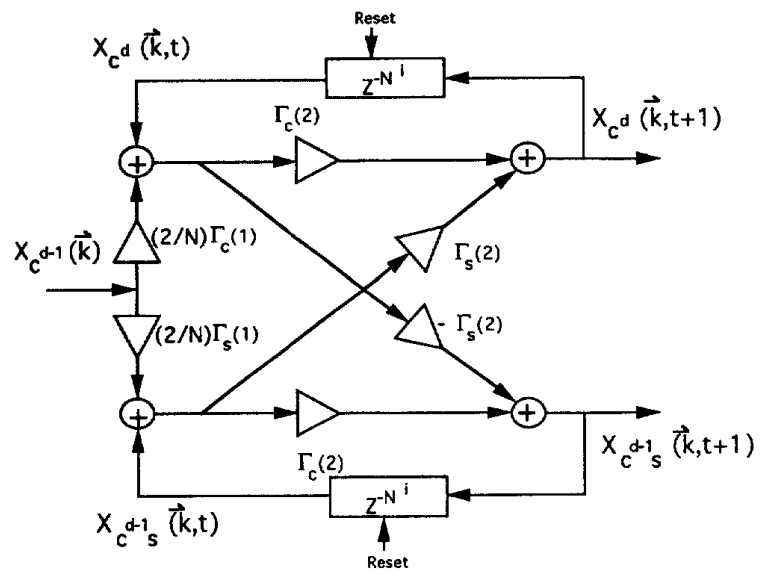

Fig. 10. The lattice module.

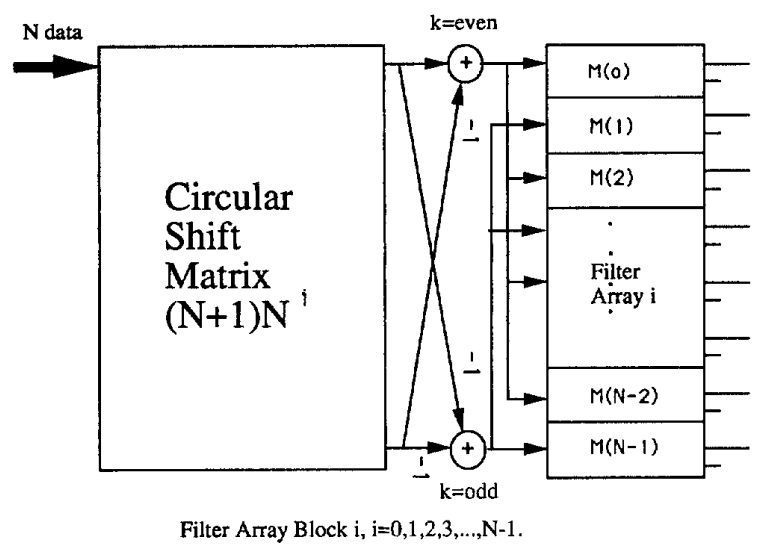

Fig. 11. The structure for Lattice Array Blocks.

DCT individually. The 3-D DCT can be implemented using either the lattice or the IIR filter structures as described below.

\section{VI.1.a. The 3-D DCT Architecture}

The architecture of the frame-recursive lattice 3-D DCT consists of three Array Blocks (AB0, AB1, and AB2) whose configurations are depicted in Fig. 11. The Array Block ABi consists of a shift register array, two adders, and a lattice or IIR array; the shift register array is of size $(N+1) \times N^{i}$ and is used to store the intermediate values. The function of the adders is to update the effect of the new data and eliminate the effect of the previous data. The structure of the lattice array is shown in Fig. 10. The difference between different lattice arrays is only in the number of delays in the feedback loop. There are $N^{i}$ delay elements in the $i$ th lattice array. For the case of the direct form implementation, the lattice array is replaced by the IIR array whose configuration, same as what in Fig. 2 except the delay in the feedback path is $z^{-N^{i}}$ instead of $z^{-1}$.

The operation of this architecture can be viewed as follows. Input data is scanned row by row and frame by frame and sent to the first module AB0 which generates the 1-D DCT of each row on every input frame. When the last datum of each row is 


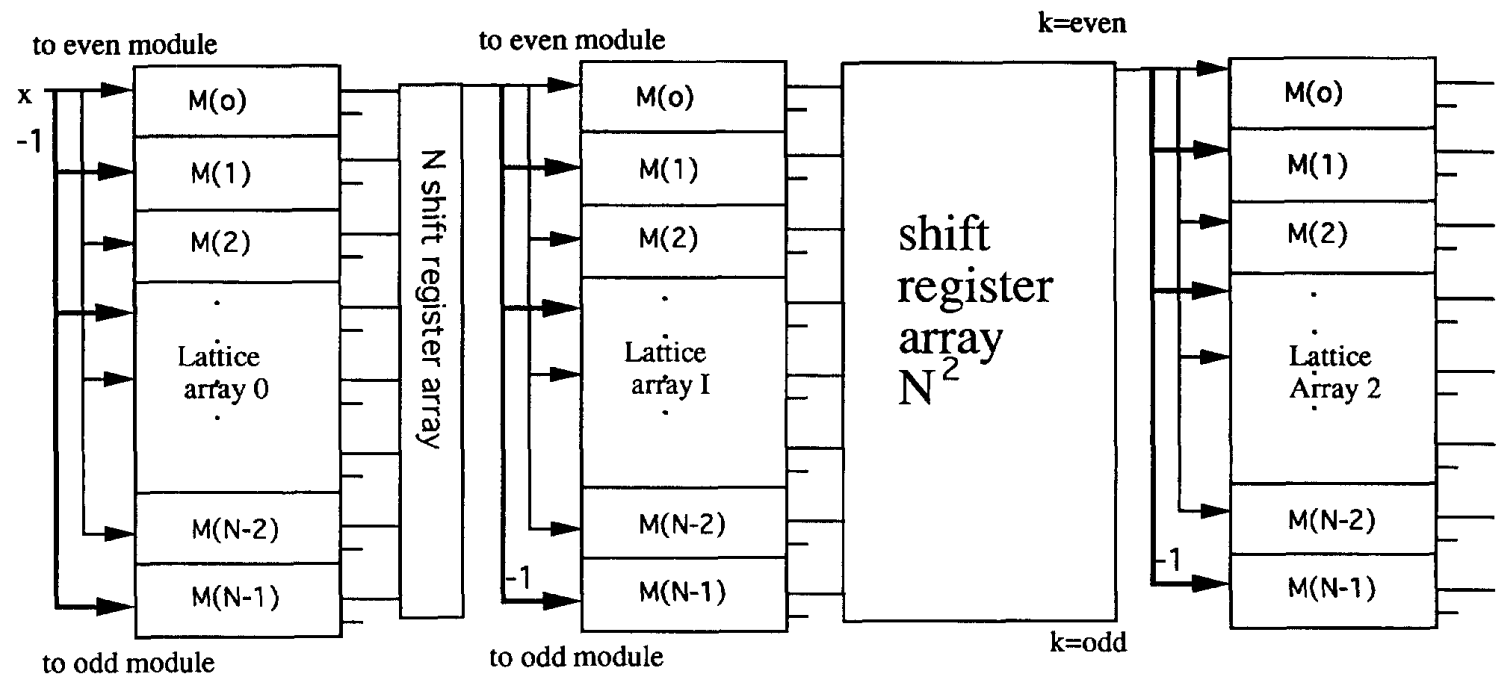

Fig. 12. The architecture for block 3-D DCT

available, the 1-D DCT of each input row vector is obtained. These $N$ 1-D DCT transformed data are loaded in parallel into the second module $\mathrm{AB} 1$ every $N$ clock cycles. The AB 1 module is used to generate the 2-D DCT of each data frame. After $N^{2}$ clock cycles, when the last datum of each frame arrives, the 2-D DCT of each frame is available. These values are loaded in parallel into the AB2 module to generate the 3-D DCT recursively. The difference between the 2-D DCT of the parity of the $(t+N)$ th and $t$ th frame is used as the input to the AB2 module. There are $N^{2}$ shift registers in the feedback loop of AB2 to store the transformed data of each frame. It takes $N^{2}$ cycles to finish updating a new 3-D block and this is the period required to obtain a new 2-D DCT data block. It is easy to verify that the system is fully-pipelined.

In applications where only block multi-dimensional transforms are required, the above architecture can be simplified. Intermediate values stored in the shift registers are not necessary. The purpose of the shift registers required is to store the current data obtained from filter arrays, hence its size is reduced to $N^{i}$ for Lattice Array Block $i$. Since the updating is unnecessary, the two adders can be eliminated. The lattice block 3-D DCT structure is shown in Fig. 12.

\section{VI.2. Time-Recursive Structures for Multi-Dimensional DXT}

In this section, we generalize the time-recursive concept to any multi-dimensional DXT and derive the fully-pipelined block structures. Denote by $\left[x\left(\vec{n}_{d}, t\right)\right]$ the input data file at time $t$, and by $\left[x\left(\vec{n}_{d}, t+1\right)\right]$ the data file at time $(t+1)$ which is obtained by shifting $\left[x\left(\vec{n}_{d}, t\right)\right]$ in a direction of one of the axes of $\vec{n}_{d}$ by one unit. For simplicity, let us assume that the data file is shifted in the direction of the last axis, $n_{d}$. The $d$-dimensional DXT of the input data file $\left[x\left(\vec{n}_{d}, t\right)\right]$ is defined as

$$
X_{X^{d}}\left(\vec{k}_{d}, t\right)=\sum_{n_{1}=0}^{N-1} \sum_{n_{2}=0}^{N-1} \cdots \sum_{n_{d}=t}^{t+N-1} x\left(\vec{n}_{d}, t\right) P_{\vec{n}_{d}}\left(\vec{k}_{d}\right),
$$

Here, we assume that the transform kernel $P_{\vec{n}_{d}}\left(\vec{k}_{d}\right)$ is separable. $^{2}$. That is

$$
P_{\vec{n}_{d}}\left(\vec{k}_{d}\right)=P_{n_{1}}\left(k_{1}\right) P_{n_{2}}\left(k_{2}\right) \cdots P_{n_{d}}\left(k_{d}\right) .
$$

From the analysis in Section VI.1, we see that the updated transform $X_{X^{d}}\left(\vec{k}_{d}, t+1\right)$ is related to the previous transform $X_{X^{d}}\left(\vec{k}_{d}, t\right)$ by the following equation [17]:

$$
\begin{aligned}
X_{X^{d}} & \left(\vec{k}_{d}, t+1\right) \\
= & \left\{X_{X^{d}}\left(\vec{k}_{d}, t\right)+X_{x^{d-1}}\right. \\
& \left.\cdot\left[\vec{k}_{d-1}, \Delta(t+N, t)\right] D_{x}(k)\right\} \Gamma_{x}(k),
\end{aligned}
$$

where $\Delta(t+N, t)$ is the difference between the data files at time $t$ and $(t+N)$, and $D_{x}(k)$ and $\Gamma_{x}(k)$ are coefficients that depend only on the transform kernel and index $k$. The above equation indicates that the $d$-dimensional DXT can be updated recursively using the previous transformed data $X_{X^{d}}\left(\vec{k}_{d}, t\right)$ and the $(d-1)$-D DXT of $\Delta(t+N, t)$. This relation can be used recursively such that any $d$-D DXT can be generated from the 1-D DXT using $d$ filter array blocks.

As described in the previous section, there are two kinds of time-recursive DXT architectures, the moving frame $d$-D DXT and the block $d$-D DXT. The structure of the basic building block in the moving-frame DXT is shown in Fig. 11, where the filter array can be either the lattice or the filter form. The function of each block is to shift the $(d-1)$-dimensional data into a data bank, then distribute the difference of the first and last frame of the data bank to the second stage DXT array. The dimension of the shift register array is $(N+1) \times N^{i}$ and the delay in filter array $i$ is $N^{i}$. The time required to obtain the $(d-1)$-dimensional DXT is $N^{d-1}$, which is also the time required to obtain the $N^{d}$ elements of the transformed data.

In the case of block DXT, the size of the shift register array can be reduced and adders can be eliminated because

\footnotetext{
${ }^{2}$ This is true for all the discrete sinusoidal transforms considered in this paper.
} 


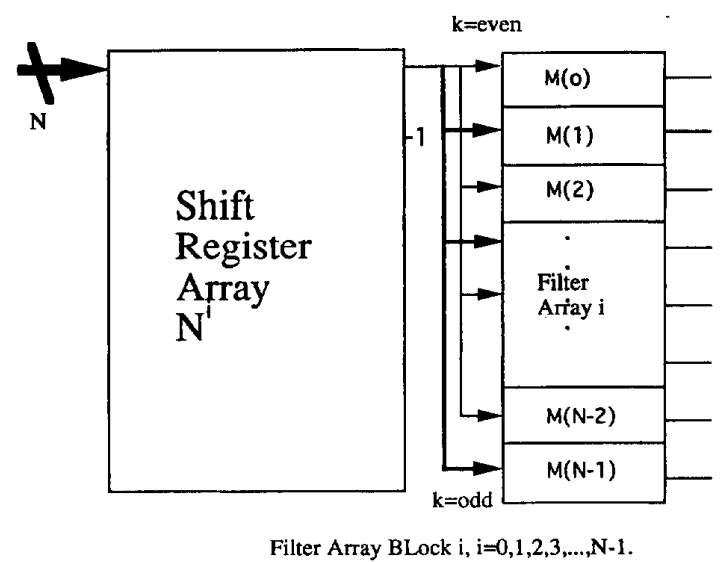

Fig. 13. The basic structure of the block DXT.

intermediate transformed data do not have to be stored. The size of the shift register array is $N^{i}$. The structure of the $L A B$ is shown in Fig. 13. The lattice array $i$ is reset every $N^{i+1}$ cycles.

\section{VI.2.a. Area-Time Complexity Analysis}

Our architecture for computing the $d$-dimensional transform DXT over $N^{d}$ points consists of $d$ blocks, each block is composed of a shift register array followed by a one-dimensional lattice or IIR structure made up of $N$ DXT modules. The $i$ th shift register array is of size $(N+1) \times N^{i} b$, where $0 \leq i \leq d-1$ and $b$ is the number of bits used to represent each number. The output is generated in a shift register array of size $N^{d} b$. Therefore the total number of multipliers and adders used is $O(d N)=O(N)$, and the total amount of memory is $O\left(N^{d} b\right)$. The next lemma states that the area of any chip that computes the $d$-dimensional DFT transform must be $\Omega\left(N^{d} b\right)$, and hence our design asymptotically optimal in its use of area. The same holds true for the remaining transforms. We are using the standard VLSI model as introduced by Thompson [30].

Lemma 6: Any VLSI system that computes the $d$ dimensional DFT on $N^{d}$ points requires area $A=\Omega\left(N^{d} b\right)$, where $b$ is the number of bits required to represent each input number.

The proof of the lemma can be derived from a result in [29] in a straightforward way. Hence our design uses the least amount of memory asymptotically. The speed of our VLSI design cannot be improved asymptotically since it processes the input in real time. Hence our design is asymptotically optimal in both speed and area.

\section{CONClusion}

In this paper, we proposed optimal time-recursive unified architectures for computing the DCT, DST, DHT, DFT, LOT, and CLT using only half as many multipliers as the unified lattice structure described in [1]. In the lattice structure, two transforms are dually generated simultaneously, while this optimal architecture has the flexibility of generating either one transform or both together. The basic configuration of the optimal unified architectures has a second order autoregressive model. It is optimal in the sense that the number of the multipliers used is minimum and both speed and area are asymptotically optimal. We also gave a theoretical justification of the unified time-recursive architecture using the Fundamental Recurrence Formula. We show that to generate the DCT and DST, only $2 N-2$ multipliers are necessary, while in the case of dual generation of the DCT and DST, only $1.5 \mathrm{~N}$ multipliers are required for each transform on average. Finally, we generalized the time-recursive concept to multi-dimensional transforms. The resulting architecture is fully-pipelined, modular, and regular. It requires only $d 1-\mathrm{D}$ arrays for computing a $d$-D DXT.

\section{REFERENCES}

[1] K. J. R. Liu and C. T. Chiu, "Unified Parallel Lattice Structures for Time-Recursive Discrete Cosine/Sine/Hartley Transforms," IEEE Trans. on Signal Processing, vol. 41, No. 3, pp. 1357-1377, March 1993.

[2] A. Rosenfeld and A. C. Kak, Digital Picture Processing, 2nd edition, Academic Press, 1982.

[3] A. V. Oppenheim and R. W. Schafer, Discrete-Time Signal Processing, Prentice Hall, 1989.

[4] P. H. Ang, P. A. Ruetz, and D. Auld, "Video compression makes big gains," IEEE Spectrum, pp. 16-19, Oct. 1991.

[5] R. Yip and K. R. Rao, "On the shift property of DCT's and DST's," IEEE Trans. Acous., Speech, Signal Processing, vol. ASSP-35, No. 3 pp. 404-406, March 1987.

[6] C. Chakrabarti and J. Jáa, "Systolic architectures for the computation of the discrete Hartley and the discrete cosine transforms based on prime factor decomposition," IEEE Trans. on Computers, vol. 39, No. 11, pp. 1359-1368, Nov. 1990

[7] W. Kou and J. W. Mark, "A new look at DCT-type transforms," IEEE Trans. Acous., Speech, Signal Processing, vol. ASSP-37, No. 12, pp. 1899-1908, Dec. 1989.

[8] N. I. Cho and S. U. Lee, "DCT algorithms for VLSI parallel implementations," IEEE Trans. Acous., Speech, Signal Processing, vol. ASSP-38, No. 1, pp. 1899-1908, Dec. 1989.

[9] L. W. Chang and M. C. Wu, "A unified systolic array for discrete cosine and sine transforms," IEEE Trans. Acous., Speech, Signal Processing, vol. ASSP-39, No. 1, pp. 192-194, Jan. 1991.

[10] Z. Wang, "Fast algorithms for the discrete $W$ transform and for the discrete Fourier transform," IEEE Trans. Acous., Speech, Signal Processing, vol. ASSP-32, Aug. 1984.

[11] N. Ahmed, T. Natarajan, and K. R. Rao, "Discrete cosine transform," IEEE Trans. Computers., vol. C-23, pp. 90-93, Jan. 1974.

[12] R. N. Bracewell, "Discrete Hartley transform," J. Opt. Soc. Amer., vol. 73, pp. 1832-1835, Dec. 1983.

[13] R. Young and N. Kingsbury, "Motion Estimation using Lapped Transforms," IEEE ICASSP Proc., pp. III 261-264, March 1992.

[14] H. S. Malvar and D. H. Staelin, "The LOT: Transform coding without blocking effects," IEEE Trans. Acous., Speech, Signal Processing, pp. 553-559, Apr. 1989

[15] A. K. Jain, Fundamentals of Digital Image Processing. Prentice-Hall, 1989.

[16] T. S. Chihara, An Introduction to Orthogonal Polynomials. Gordon and Breach, 1978.

[17] C. T. Chiu and K. J. R. Liu, "Real-Time Parallel and Fully-Pipelined Two-Dimensional DCT Lattice Structures with Application to HDTV Systems," IEEE Trans. on Circuits and Systems for Video Technology, pp. 25-37, March 1992.

[18] K. J. R. Liu, "Novel parallel architectures for short-time Fourier transform," to appear, IEEE Trans. on Circuits and Systems-II: Analog and Digital Signal Processing, Sept. 1993.

[19] K. J. R. Liu, C. T. Chiu, R. Kolagotla, and J. JáJá, "Optimal unified IIR architectures for time-recursive discrete sinusoidal transforms," Proc. IEEE Int'l Conf. Acoustic, Speech, and Signal Processing (ICASSP) pp. III-73-76, Minneapolis, April 1993.

[20] H. S. Hou, "A fast recursive algorithm for computing the discrete cosine transform," IEEE Trans. Acous., Speech, Signal Processing, vol. ASSP-35, pp. 1455-1461, Oct. 1987.

[21] R. N. Bracewell, "The Fast Hartley transform," Proc. IEEE, vol. 72, pp. 1010-1018, Aug. 1984 
[22] W. H. Chen, C. H. Smith, and S. C. Fralick, "A fast computational algorithm for the discrete cosine transform," IEEE Trans. Communication. vol. COM-25, pp. 1004-1009, Sept. 1977.

[23] M. Vetterli and H. Nussbaumer, "Simple FFT and DCT algorithm with reduced number of operations," Signal Processing, vol. 6, no. 4, pp. 267-278, Aug. 1984.

[24] H. W. Jones, D. N. Hein, and S. C. Knauer, "The Karhunen-Loeve, discrete cosine and related transform via the Hadmard transform," in Proc. Inc. Telemeter, Conf., Los Angeles, CA, pp. 87-98, Nov. 1978.

[25] B. G. Lee, "A new algorithm to compute the discrete cosine transform," IEEE Trans. Acous., Speech, Signal Processing, vol. ASSP-32, pp 1243-1245, Dec. 1984

[26] H. S. Hou, "The fast Hartley transform algorithm," IEEE Trans, on Computers, vol. C-36, No. 2, pp. 147-156, Feb. 1987.

[27] H. V. Sorenson et al., "On computing the discrete Hartley transform," IEEE Trans. Acous., Speech, Signal Processing, vol. ASSP-33, No. 4, pp. 1231-1238, Oct. 1985

[28] S. B. Narayanan and K. M. M. Prabhu, "Fast Hartley transform pruning," IEEE Trans. Acous., Speech, Signal Processing, vol. ASSP-39, No. 1, pp. 230-233, Jan. 1991.

[29] P. Duris et al. "Tight chip area lower bounds for discrete Fourier and Walsh-Hadamard transformations," Information Processing Letters 21 , pp. $245-247,1985$.

[30] C. D. Thompson, "A complexity theory for VLSI," Ph.D. Thesis, Department of Computer Science, Carnegie Mellon University, Pittsburgh, 1980.

[31] C. T. Chiu, R. Kolagotla, K. J. R. Liu, and J. JáJá, "VLSI implementation of real-time parallel DCT/DST lattice structure for video communications," in VLSI Signal Processing V, Ed. Yao, Jain, Przytula, and Rabaey, pp. 101-110, IEEE Press, 1992.

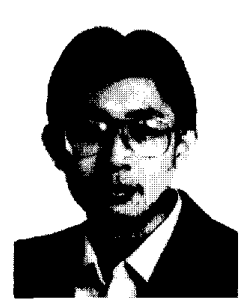

K. J. Ray Liu (S'86-M'90-SM'93) received the B.S. degree in electrical engineering from National Taiwan University in 1983, the M.S.E. degree in electrical engineering and computer science from the University of Michigan, Ann Arbor in 1987, and the Ph.D. degree in electrical engineering trom the University of California, Los Angeles, in 1990.

During 1983-1985, he served in the Signal Corps. Taiwan, as a Communications Officer. He then became a Teaching and Research Assistant at the University of Michigan and the University of California Los Angeles. Since 1990, he has been an Assistant Professor of Electrical Engineering Department and Institute for Systems Research, University of Maryland, College Park. His research interests span all the aspects of high performance signal processing including parallel and distributed processing, fast algorithm, VLSI, and concurrent architecture, with application to image/video, radar/sonar, communications, and medical and biomedical technology.

Dr. Liu received the IEEE Signal Processing Society's 1993 Senior Award. He was awarded the Research Initiation Award from the National Science Foundation, the President Research Partnership from the University of Michigan, and the University Fellowship and the Hortense Fishbaugh Memorial Scholarship from the UCLA. He was also awarded the Achievement Award in Science and Engineering from the Taiwanese-American Foundation. Dr. Liu is a member of VLSI Signal Processing Technical Committee of the IEEE Signal Processing Society. He is also a member of ACM and SIAM.

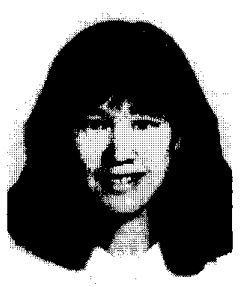

Ching-Te Chiu (S'90-M'93) received the B.S. and M.S. degree in electrical engineering from National Taiwan University, Taiwan, in 1986 and 1988, and the Ph.D. degree in electrical engineering from University of Maryland, College Park in 1992.

Her research experience includes as a summer research student at Electronics Research Service Organization (ERSO), National Taiwan Institute of Technology in 1987. From 1989 to 1992, she was a research assistant in electrical engineering at the University of Maryland, College Park. In 1993, she joined National Chung Cheng University in Taiwan, where she is an associate professor of Electrical Engineering Department. Her research interests include signal processing, multidimensional signal processing. VLSI algorithms and architectures, VLSI fault-tolerance, image processing and HDTV systems.

Dr. Chiu is a member of the IEEE Signal Processing Society. She received the dissertation fellowship from University of Maryland in 1992.

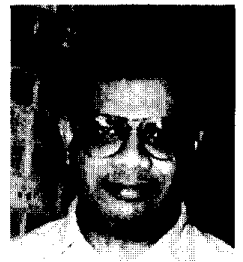

Ravi K. Kolagotla (S'86-M'86-M'92)received his B.Tech. degree from the Indian Institute of Technology in 1985, his M.S. degree from Rensselaer Polytechnic Institute in 1987, and his Ph.D. degree from the University of Maryland in 1992, all in Electrical Engineering.

He joined IBM Corp., Essex Jn., VT in 1992, where he is currently working on BiCMOS products. His research interests include VLSI architectures and algorithms, data compression, and image processing.

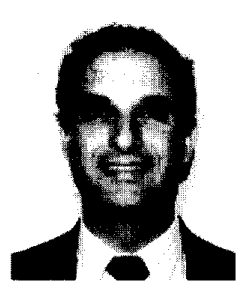

Joseph F. JáJá (SM'88) received the Ph.D. degree in Applied Mathematics from Harvard University in 1977. He is currently a Professor of Electrical Engineering, Institute For Advanced Computer Studies, and Institute For Systems Research at the Univer sity of Maryland, College Park. He has published numerous papers on paraliel algorithms, VLSI signal processing, algebraic complexity, and computational complexity. He is the author of the book, An Introduction to Parallel Algorithms, published by Addison-Wesley, 1992, and he is a Subject Computing. 